\title{
Hypertrophied Right Inferior Phrenic Artery in Cirrhotic Patients without Hepatocellular Carcinoma: An Interesting Observation on 256 Slice Multidetector Computed Tomography
}

\author{
Chinmay Bhimaji Kulkarni¹,๑ P. K. Nazar ${ }^{1} \quad$ Sreekumar Karumathil Pullara ${ }^{1} \quad$ Nirmal Kumar Prabhu ${ }^{1}$ \\ Srikanth Moorthy ${ }^{1}$
}

1Department of Radiology, Amrita Institute of Medical Sciences,

Address for correspondence Chinmay Bhimaji Kulkarni, Associate Amrita Vishwa Vidyapeetham, Cochin, Kerala, India \begin{abstract}
Professor, Department of Radiology, Amrita Institute of Medical Sciences and Research Center, AIMS, Kochi- 682041, Kerala, India (e-mail: drchinmaykulkarni@gmail.com, chidoc@rediffmail.com).
\end{abstract}

\begin{abstract}
Keywords

- aorta

- cirrhosis

- portal hypertension

- multidetector computed tomography

- inferior phrenic artery

- celiac artery

- chemoembolization

Aim To evaluate whether right inferior phrenic artery (RIPA) is a source of extrahepatic arterial supply to the liver in cirrhotic patients without hepatocellular carcinoma (HCC) using 256 slice computed tomography (CT).

Materials and Methods Institutional review board approval was obtained for this retrospective study. A total of 262 consecutive cirrhotic patients (male: female-172:90; mean age $56.45 \pm 12.96$ years) without HCC and hepatic vascular invasion, and who underwent technically successful multiphase CT, were included in the study. Additionally, 280 noncirrhotic patients (male:female-169:111; mean age $54.56 \pm 14.21$ years) who underwent abdominal multiphase CT scans for indications other than liver disease and did not have focal liver lesions or hepatic vascular disease were included as a control group. The RIPA and left inferior phrenic artery (LIPA) diameters were measured at the level of the ascending segment of IPA located anterior to the diaphragmatic crus. The relationship between RIPA diameters and Child-Pugh score was assessed.

Results The cirrhotic patient group and control group were matched for age $(p=0.11)$ and gender $(p=0.20)$. The mean diameter of RIPA in the cirrhotic group $(1.93 \pm 0.4 \mathrm{~mm})$ was significantly higher than in the control group $(1.50 \pm 0.5 \mathrm{~mm})$, $p<0.001$. The mean diameter of LIPA in the cirrhotic group $(1.34 \pm 0.5 \mathrm{~mm})$ was not significantly higher than in the control group $(1.30 \pm 0.5 \mathrm{~mm}), p=0.32$. We found a statistically linear and moderate degree relationship between RIPA diameter values and Child-Pugh scores ( $p=0.002, r=0.593)$.

Conclusion RIPA is hypertrophied in patients with cirrhosis without HCC. It may be an important contributor to the blood flow to the liver in cirrhotic patients even without HCC, especially with portal hypertension.
\end{abstract}

DOI https://doi.org/

$10.1055 / \mathrm{s}-0040-1721530$

ISSN 2457-0214.
(C)2020. Indian Society of Vascular and Interventional Radiology. This is an open access article published by Thieme under the terms of the Creative Commons Attribution-NonDerivative-NonCommercial-License, permitting copying and reproduction so long as the original work is given appropriate credit. Contents may not be used for commercial purposes, or adapted, remixed, transformed or built upon. (https://creativecommons.org/licenses/by-nc-nd/4.0/).

Thieme Medical and Scientific Publishers Pvt. Ltd. A-12, 2nd Floor, Sector 2, Noida-201301 UP, India 


\section{Introduction}

The right inferior phrenic artery (RIPA) is the most common extrahepatic blood supply to hepatocellular carcinoma (HCC), especially those located near the bare area of the liver and posteriorly located exophytic lesions. It is also observed that in patients with stenosis/occlusion of hepatic arteries after repeated transarterial chemoembolization (TACE), HCCs commonly recruit blood supply from RIPA. Identifying and targeting these branches while performing TACE is essential for the effective outcome. ${ }^{1-3}$ Studies have shown that RIPA is a common extrahepatic artery to supply liver when the hepatic arteries are ligated/occluded. ${ }^{4-6}$ It is established that in cirrhotic patients with portal hypertension, which is a state of restricted blood supply to the liver, hepatic arteries enlarge and supply the liver. ${ }^{7-11}$ However, the literature on the extrahepatic blood supply through RIPA to the cirrhotic liver without HCC is limited. Understanding the anatomical variations and physiology of RIPA will help us in better management of cirrhotic patients. In this study, we have tried to evaluate whether RIPA has a role in supplying the cirrhotic liver without HCC.

\section{Aim}

To evaluate whether RIPA is a source of extrahepatic arterial supply to the liver in cirrhotic patients without HCC.

\section{Materials and Methods}

\section{Study Population}

An institutional review board approved this retrospective study. Informed consent was taken before the CT scans. Between January 2018 to November 2019, 262 consecutive cirrhotic patients (male: female-172:90; mean age $56.45 \pm$ 12.96 years) who underwent technically successful multiphase CT for evaluation of liver were included in the study. None of the patients included in the study had primary or metastatic hepatic neoplasm at the time of the CT scan. Also, none of the patients had signs of hepatic vascular disease, pleural or diaphragmatic disease. The diagnosis of cirrhosis was made by a combination of imaging (ultrasonography, CT, upper endoscopic) and laboratory tests. CT findings of collaterals, ascites, splenomegaly, and dilated portal (>13 mm) were considered as evidence of portal hypertension. The Child-Pugh score of the patients was calculated by using the clinical and laboratory database at the time of the CT examination. In addition, 280 noncirrhotic patients (male: female-169:111; mean age $54.56 \pm 14.21$ years) who underwent abdominal multiphase $\mathrm{CT}$ scans for indications other than liver disease (mesenteric ischemia, peripheral arterial disease, donor patients for renal transplantation, renal artery stenosis, and aortic aneurysms), and did not have focal liver lesions or hepatic vascular disease, pleural or diaphragmatic disease, were included as control group. Patients with known HCC and portal vein thrombosis, and those who had undergone endovascular treatment of aorta, were excluded from the study. CT images of patients who had severe atherosclerosis, and/or movement artifacts preventing assessment of the IPA, were also excluded from the study.

\section{CT Technique}

Multidetector CT (MDCT) was performed with a 256-section multidetector CT scanner (Philips 256 slice ICT, Philips Healthcare). Scanning parameters are shown in - Table 1. Contrast-enhanced images were obtained in the bolustriggered arterial phase and delayed venous phases. Patients were scanned at $120 \mathrm{kV}$ with an active dose reduction index (DRI-20).

\section{Image Interpretation}

The image analysis was done on dedicated workstations (Intellspace, Philips Healthcare) which allowed three-dimensional (3D) postprocessing with multiplanar image reformatting and maximum intensity projections (MIPs). Arterial phase CT images with $1 \mathrm{~mm}$ slice thickness were used to assess the IPA. The IPA diameters were measured with electronic calipers at the level of the ascending segment of IPA located anterior to the diaphragmatic crus on axial CT images. All images were analyzed by a radiologist with 10 years of experience in abdominal radiology. The relationship between RIPA diameters and the Child-Pugh score was assessed.

\section{Statistical Analysis}

Statistical analysis was performed using IBM SPSS Statistics 20.0 software (IBM Corp.). The data was analyzed using descriptive statistical methods. Quantitative variables were expressed as mean values and standard deviations (SDs). The gender distribution among the cirrhotic and control groups was compared by the chi-square test. The mean age difference between the cirrhotic and control groups was assessed by Student's $t$-test. Statistical significance was

Table 1 Multidetector CT parameters used for scanning patients

\begin{tabular}{|l|l|}
\hline Parameter & Comment \\
\hline Peak voltage (kVp) & $120 \mathrm{kVP}$ \\
\hline Effective (mAs) & $150-350 \mathrm{mAs}$ (DRI-20) \\
\hline Rotation time (s) & $0.5 \mathrm{~s}$ \\
\hline Detector collimation & $128 \times 0.625 \mathrm{~mm}$ \\
\hline Pitch & 0.914 \\
\hline Matrix & $512 \times 512$ \\
\hline $\begin{array}{l}\text { Reconstruction slice } \\
\text { thickness }\end{array}$ & $1.0 \mathrm{~mm}$ \\
\hline Reconstruction method & iDose-4 \\
\hline IV Contrast: & $\begin{array}{l}\text { Omnipaqu 350 (iohexol); volume: } \\
1.5 \mathrm{~mL} / \mathrm{kg} ; \text { rate: 4-6 mL/sec }\end{array}$ \\
\hline Scan delay & $\begin{array}{l}\text { Hepatic AP: 15 sec; Other AP: 6 sec } \\
\text { (Bolus tracking; ROl: descending } \\
\text { aorta, } 2 \text { cm above diaphragm) }\end{array}$ \\
\hline
\end{tabular}

Abbreviations: AP, arterial phase; HU, Hounsfield; ROI, region of interest. 
established at a $p$-value of less than 0.05. The linear relationship between the Child-Pugh score and RIPA diameter was calculated by Pearson's correlation coefficient.

\section{Results}

The patient group consisted of 262 cirrhotic patients (male: female-172:90; mean age $56.45 \pm 12.9$ years) and the control group consisted of 280 patients (male:female-169:111; mean age $54.56 \pm 14.2$ years) with no liver, pleural, or diaphragmatic disease. There was no statistically significant difference between the two groups with regard to age and gender ( $p=0.11$, and $p=0.20$, respectively) (-Table 2 ). The origins of the IPAs were visualized in all patients. The origins of the IPAs from various sources with or without common truncus in both the patient and control groups are shown in - Table 3. The mean diameter of RIPA in the cirrhotic group $(1.93 \pm 0.4 \mathrm{~mm})$ was significantly higher than in the control group ( $1.50 \pm 0.5 \mathrm{~mm}), p<0.001$. In the cirrhotic group, the diameter of the RIPA $(1.93 \pm 0.4 \mathrm{~mm})$ was significantly higher than the LIPA diameter $(1.34 \pm 0.5), p<0.001$ (-Figs. 1 and 2 ). There was no statistically significant difference in the diameter of the LIPA between the patient group $(1.34 \pm 0.5 \mathrm{~mm})$ and the control group $(1.3 \pm 0.5 \mathrm{~mm}), p=0.32$. Even in the control group, although the RIPA diameter $(1.50 \pm 0.5 \mathrm{~mm})$ was higher compared with LIPA diameter $(1.3 \pm 0.5 \mathrm{~mm})$, this was not statistically significant ( $p=0.14)$.

The cause of the cirrhosis was ethanol-related in 123 patients (46.9\%), hepatitis B in 55 patients (20.9\%), hepatitis C in 47 patients (17.9\%), cryptogenic in 27 patients (10.3\%), autoimmune in seven patients $(2.7 \%)$, and primary biliary cirrhosis in three patients (1.1\%). According to the ChildPugh classification, 82 patients (31.3\%) were in class A, 123 patients (46.9\%) were in class B, and 57 patients (21.8\%) were in class $\mathrm{C}$. The mean value of the Child-Pugh score was $8.13 \pm$ 2.14 (range: 5-14). We found a statistically linear and moderate degree relationship between RIPA diameter values and Child-Pugh scores ( $p=0.002, r=0.593)$ ( -Fig. 3 ).

\section{Discussion}

The liver receives blood supply from both portal vein (75\%) and hepatic artery (25\%). ${ }^{12-15}$ In conditions leading to the

Table 2 Characteristics of patients in cirrhotic and the control group

\begin{tabular}{|l|l|l|l|}
\hline & $\begin{array}{l}\text { Cirrhosis } \\
(N=262)\end{array}$ & $\begin{array}{l}\text { Control } \\
(N=280)\end{array}$ & $p$-Value \\
\hline Age (years) & $56.45 \pm 12.9$ & $54.56 \pm 14.2$ & 0.11 \\
\hline Sex & & & \\
\cline { 1 - 3 } Male $(N=341)$ & $172(50.4 \%)$ & $169(49.6 \%)$ & \multirow{2}{*}{0.20} \\
\cline { 1 - 3 } Female $(N=201)$ & $90(44.8 \%)$ & $111(55.2 \%)$ & \multirow{2}{*}{$<0.001$} \\
\hline Diameter of RIPA & $1.93 \pm 0.4$ & $1.50 \pm 0.5$ & 0.32 \\
\hline Diameter of LIPA & $1.34 \pm 0.5$ & $1.3 \pm 0.5$ & \multirow{2}{|l|}{0.5} \\
\hline
\end{tabular}

Abbreviations: LIPA, left inferior phrenic artery; RIPA, right inferior phrenic artery. decreased blood flow to the liver, extrahepatic pathways open up to supply the liver. In cirrhosis, the liver is in a state of relative ischemia once portal hypertension sets in. The most common pathway of compensating liver blood flow is through the hypertrophied hepatic artery. ${ }^{11,16,17}$ Along with

Table 3 Variations in the origins of RIPA and LIPA in control and cirrhotic groups

\begin{tabular}{|c|c|c|}
\hline \multicolumn{3}{|l|}{ Control group $(N=280)$} \\
\hline Common origin & \multicolumn{2}{|c|}{ RIPA + LIPA } \\
\hline Aorta & \multicolumn{2}{|l|}{30} \\
\hline Celiac & \multicolumn{2}{|l|}{29} \\
\hline Left renal artery & \multicolumn{2}{|l|}{1} \\
\hline Separate origin & RIPA & LIPA \\
\hline Aorta & 99 & 78 \\
\hline Celiac artery & 82 & 135 \\
\hline Right renal artery & 34 & 0 \\
\hline Left renal artery & 0 & 3 \\
\hline Left gastric artery & 4 & 0 \\
\hline Splenic artery & 0 & 4 \\
\hline SMA & 1 & 0 \\
\hline \multicolumn{3}{|l|}{ Cirrhotic group $(N=262)$} \\
\hline Common origin & \multicolumn{2}{|c|}{ RIPA + LIPA } \\
\hline Aorta & \multicolumn{2}{|l|}{33} \\
\hline Celiac & \multicolumn{2}{|l|}{22} \\
\hline Separate origin & RIPA & LIPA \\
\hline Aorta & 105 & 79 \\
\hline Celiac & 78 & 125 \\
\hline Right renal artery & 20 & 0 \\
\hline Splenic artery & 0 & 2 \\
\hline Left renal artery & 0 & 1 \\
\hline Left gastric artery & 3 & 0 \\
\hline Common hepatic artery & 1 & 0 \\
\hline
\end{tabular}

Abbreviations: LIPA, left inferior phrenic artery; RIPA, right inferior phrenic artery; SMA, superior mesenteric artery.

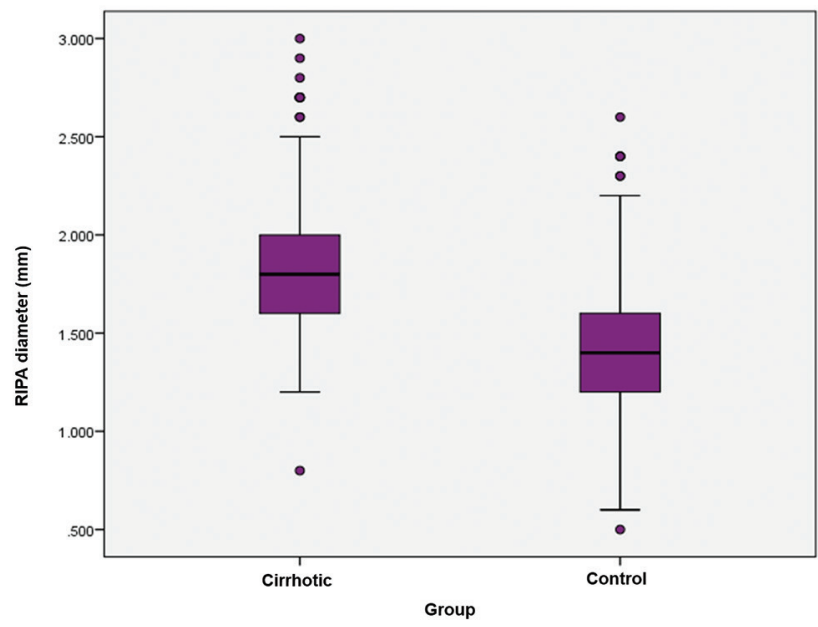

Fig. 1 Box-and-whisker diagram showing the diameters of the right inferior phrenic artery (RIPA). 


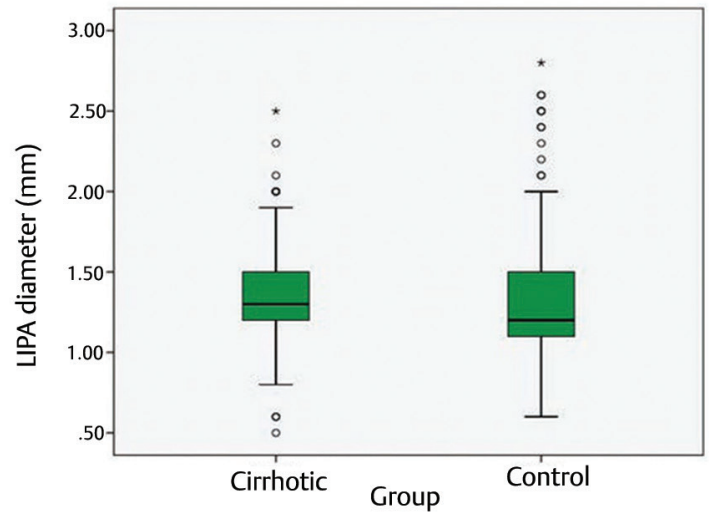

Fig. 2 Box-and-whisker diagram showing the diameters of the left inferior phrenic artery (LIPA).

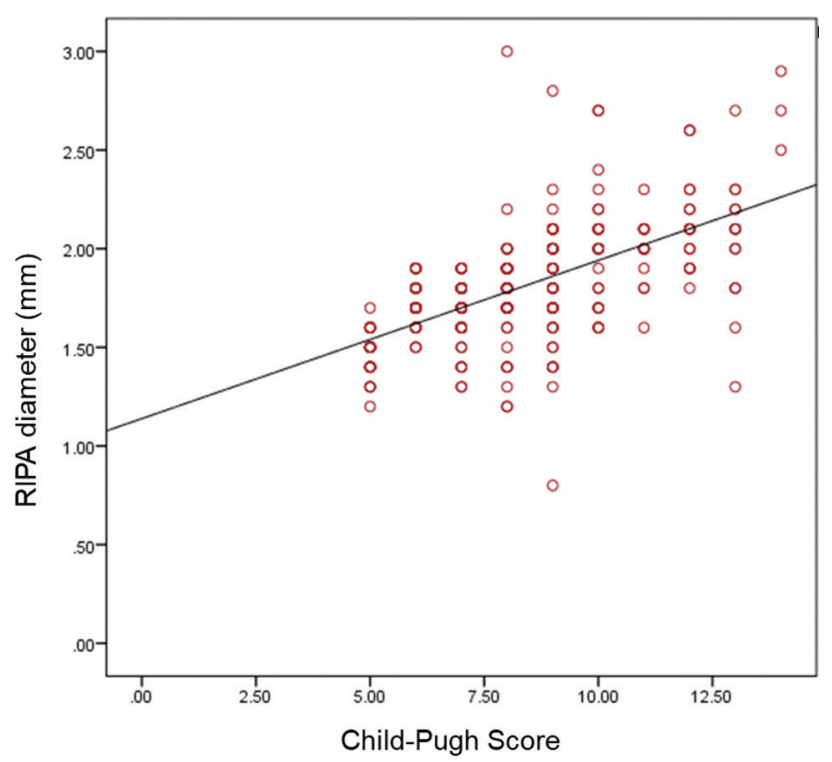

Fig. 3 Relationship between Child-Pugh scores and right inferior phrenic artery (RIPA) diameters.

the hepatic artery, other extrahepatic collateral pathways also supply the liver. RIPA is the most common extrahepatic collateral blood flow to the HCC.1-3 In this study, we have tried to understand the role of RIPA in supplying cirrhotic livers without HCC.

In cirrhosis, parenchymal fibrosis affects the hepatic sinusoids, leading to increased resistance to the portal blood flow and resulting in portal hypertension. As the cirrhosis progresses, portal venous blood flow to liver decreases, and in later stages, reversal of portal venous flow may occur. ${ }^{18-21}$ Communication between the portal vein and hepatic artery is through trans-sinusoidal, transvasal, and transbiliary vascular plexus route. As the portal venous blood flow to the liver decreases, hepatic arteries enlarge to compensate the blood supply to the liver. ${ }^{22-24}$ In a study by Toni et al, the mean diameters of the splenic and hepatic arteries were larger in cirrhotic patients. ${ }^{25}$ Takeuchi et al studied the potential routes of extrahepatic arterial supply to the liver after temporary balloon occlusion of the proper hepatic artery. They found that the commonest source of the extrahepatic arterial supply to the liver during temporary balloon occlusion of the proper hepatic artery was RIPA. ${ }^{4}$ Esen et al had studied the influence of portal hypertension on the RIPA diameter and had concluded that portal hypertension is an important triggering factor for the enlargement of RIPA. They found that the mean diameter of the RIPA in the cirrhotic group was significantly greater than the control group. In the cirrhotic group, they also found a linear and moderate relationship between RIPA diameters and Child-Pugh scores $(p=0.012, r=0.405) .{ }^{26}$ In our study, the mean RIPA diameter was significantly higher in the cirrhotic group ( $1.93 \pm 0.4 \mathrm{~mm}$ ) than the control group $(1.50 \pm 0.5 \mathrm{~mm}$ ) with a $p$ value of $<0.001$ (-Figs. 4 and $\mathbf{5}$ ). We also found a linear and moderate degree relationship between the RIPA diameter and the Child-Pugh scores $(p=0.002, r=0.593)$. Ascites and encephalopathy, the two major components of the Child-Pugh scoring system, are manifestations of portal hypertension. Ramanathan et al in their study have shown a good correlation between the
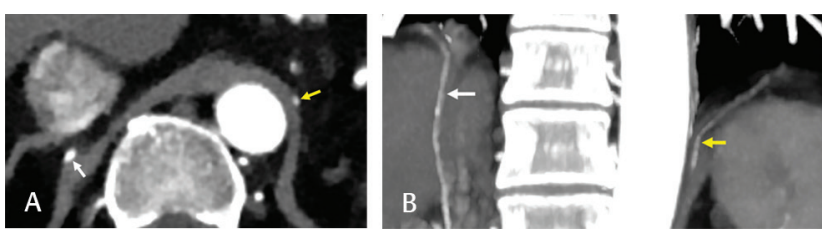

Fig. 4 A 56-year-old cirrhotic patient with Child-Pugh B status. Multidetector CT arterial phase MIP images show right IPA (A, B white arrow) and left IPA (A, B yellow arrow). Note the hypertrophied RIPA. IPA, inferior phrenic artery; MIP, maximum intensity projection; RIPA, right inferior phrenic artery.

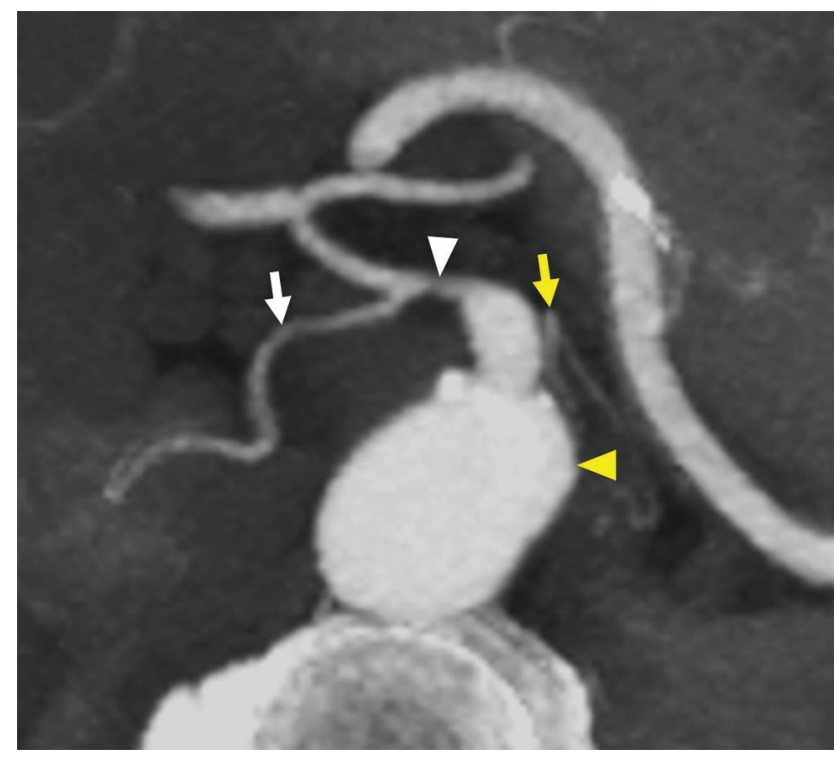

Fig. 5 A 62-year-old cirrhotic patient with Child-Pugh B status. Multidetector CT arterial phase MIP images show RIPA (white arrow) and LIPA (yellow arrow) originating separately without common truncus. RIPA originates from left gastric artery (white arrowhead) and LIPA from aorta (yellow arrowhead). Note the hypertrophied RIPA. LIPA, left inferior phrenic artery; MIP, maximum intensity projection; RIPA, right inferior phrenic artery. 
hepatic venous pressure gradient (HVPG) levels and the Child-Pugh scores of the patients. ${ }^{27}$ The Child-Pugh score seems to be an indirect indicator of the portal pressure. Hence, enlarged RIPA could serve as a surrogate marker for portal hypertension. The retrospective nature of our study did not allow us to measure HVPG in our patients. In another study, Ozbulbul et al observed that the diameter of RIPA was greater in a subgroup of patients with cirrhosis and portal vein thrombosis as compared with patients with cirrhosis without portal vein thrombosis. ${ }^{28}$ In our study, none of the patients had portal vein thrombosis. However, portal vein thrombosis is a complication of advanced portal hypertension and hence their observation is supportive of our findings. We found no statistically significant differences in the diameters of the LIPA between the cirrhotic group (1.34 \pm 0.5$)$ and the control group $(1.30 \pm 0.5), p=0.32$. This reinforces the fact that RIPA is the critical artery supplying the liver when its blood flow is compromised. However, LIPA is an important extrahepatic collateral artery supplying the tumors located in the left lobe of the liver abutting the diaphragm..$^{2,3}$ In our control group, the RIPA diameter was larger $(1.50 \pm 0.5 \mathrm{~mm})$ compared with LIPA diameter $(1.3 \pm 0.5 \mathrm{~mm})$ without any statistically significant difference $(p=0.14)$. This finding is similar to the observation of Ozbulul et al who also found the RIPA dominance in their control group..$^{28}$

Various other clinical conditions leading to the decreased arterial flow to the liver may induce extrahepatic blood supply pathways to the liver. This is especially important when we treat the HCC with TACE. Hyo-Cheol Kim et al had shown that repeated chemoembolization sessions and dorsal location of the tumors are significant factors that influence the parasitic supply of the tumor by RIPA. ${ }^{29}$ Repeated TACE induces a state of ischemia which, in turn, may lead to hypertrophy of the RIPA and supply of tumor. Even in patients with a patent hepatic artery, the RIPA is the most common extrahepatic blood supply to HCC, especially those located in the bare area of the liver and posteriorly located exophytic lesions. ${ }^{1-3}$

Our study had a few limitations. We did not perform the HVPG and relied on the CT findings and Child-Pugh score as indirect indicators of portal hypertension. The retrospective nature of the study did not allow us to measure the HVPG. We did not compare diameter measurements with conventional angiography. Moreover, the small absolute measurements of the IPAs may be a challenge in terms of repeatability, and automated techniques may be more reproducible.

\section{Conclusion}

The positive correlation between Child-Pugh scores and RIPA hypertrophy indicates that an increasing percentage of liver blood flow, although presumably small in absolute terms, is supplied by this artery as cirrhosis advances. The concept of RIPA having a physiological role is cirrhosis, apart from being a supplementary source of blood supply for HCC, could have a bearing on clinical decision-making in patients undergoing TACE. Simultaneous embolization of the hepatic artery and the RIPA in patients with advanced Child B cirrhosis with HCC could compromise liver function, and a strategy of sequential embolization over two sittings may be more appropriate. However, a randomized control trial (RCT) or a large retrospective study would be required to establish an association with post-TACE liver dysfunction and simultaneous RIPA/hepatic artery embolization.

\section{Conflicts of Interest}

The authors declare that they have no conflicts of interest.

\section{Acknowledgment}

We would like to acknowledge the invaluable advice and support of our colleagues in the Department of Anatomy and Department of Bio-Statistics, Amrita Institute of Medical Sciences. This work would not have been possible without the dedication and skill of our radiology technicians and nursing staff.

\section{REFERENCES}

1 Chung JW, Park JH, Han JK, Choi BI, Kim TK, Han MC. Transcatheter oily chemoembolization of the inferior phrenic artery in hepatocellular carcinoma: the safety and potential therapeutic role. J Vasc Interv Radiol 1998;9(3):495-500

2 Kim HC, Chung JW, Lee W, Jae HJ, Park JH. Recognizing extrahepatic collateral vessels that supply hepatocellular carcinoma to avoid complications of transcatheter arterial chemoembolization. Radiographics 2005;25(Suppl 1) :S25-S39

3 Miyayama S, Matsui O, Taki K, et al. Extrahepatic blood supply to hepatocellular carcinoma: angiographic demonstration and transcatheter arterial chemoembolization. Cardiovasc Intervent Radiol 2006;29(1):39-48

4 Takeuchi Y, Arai Y, Inaba Y, Ohno K, Maeda T, Itai Y. Extrahepatic arterial supply to the liver: observation with a unified CT and angiography system during temporary balloon occlusion of the proper hepatic artery. Radiology 1998;209(1):121-128

5 Gokan T, Hashimoto T, Matsui S, Kushihashi T, Nobusawa H, Munechika H. Helical CT demonstration of dilated right inferior phrenic arteries as extrahepatic collateral arteries of hepatocellular carcinomas. J Comput Assist Tomogr 2001;25(1):68-73

6 Okino Y, Kiyosue H, Matsumoto S, Takaji R, Yamada Y, Mori H. Hepatocellular carcinoma: prediction of blood supply from right inferior phrenic artery by multiphasic CT. J Comput Assist Tomogr 2003;27(3):341-346

7 Burton-Opitz R. The vascularity of the liver: the influence of the portal blood flow upon the flow in the hepatic artery. QJ Exp Physiol 1911;4:93-102

8 Lautt WW, Role and control of the hepatic artery. In: Lautt WW, ed. Hepatic Circulation in Health and Disease. New York: Raven Press; 1981:203-226

9 Lautt WW, Legare DJ, Ezzat WR. Quantitation of the hepatic arterial buffer response to graded changes in portal blood flow. Gastroenterology 1990;98(4):1024-1028

10 Jakab F, Ráth Z, Schmal F, Nagy P, Faller J. The interaction between hepatic arterial and portal venous blood flows; simultaneous measurement by transit time ultrasonic volume flowmetry. Hepatogastroenterology 1995;42(1):18-21

11 Kreuer S, Elgethun M, Tommack M. Imaging findings of cirrhosis. J Am Osteopath Coll Radiol 2016;5:5-13

12 Vollmar B, Menger MD. The hepatic microcirculation: mechanistic contributions and therapeutic targets in liver injury and repair. Physiol Rev 2009;89(4):1269-1339

13 Schenk WG, Jr, McDonald JC, Mcdonald K, Drapanas T. Direct measurement of hepatic blood flow in surgical patients: with related observations on hepatic flow dynamics in experimental animals. Ann Surg 1962;156:463-471 
14 Rappaport AM. Hepatic blood flow: morphologic aspects and physiologic regulation. Int Rev Physiol 1980;21:1-63

15 Greenway CV, Stark RD. Hepatic vascular bed. Physiol Rev 1971;51(1):23-65

16 Bosch J, García-Pagán JC. Complications of cirrhosis. I. Portal hypertension. J Hepatol 2000;32(1, Suppl):141-156

17 Wachsberg RH, Bahramipour P, Sofocleous CT, Barone A. Hepatofugal flow in the portal venous system: pathophysiology, imaging findings, and diagnostic pitfalls. Radiographics 2002;22(1):123-140

18 La Mura V, Nicolini A, Tosetti G, Primignani M. Cirrhosis and portal hypertension: The importance of risk stratification, the role of hepatic venous pressure gradient measurement. World J Hepatol 2015;7(4):688-695

19 Garcia-Tsao G, Friedman S, Iredale J, Pinzani M. Now there are many (stages) where before there was one: In search of a pathophysiological classification of cirrhosis. Hepatology 2010;51(4):1445-1449

20 Brown JJ, Naylor MJ, Yagan N. Imaging of hepatic cirrhosis. Radiology 1997;202(1):1-16

21 D'Amico G, Garcia-Tsao G, Pagliaro L. Natural history and prognostic indicators of survival in cirrhosis: a systematic review of 118 studies. J Hepatol 2006;44(1):217-231

22 Kito Y, Nagino M, Nimura Y. Doppler sonography of hepatic arterial blood flow velocity after percutaneous transhepatic portal vein embolization. AJR Am J Roentgenol 2001;176(4):909-912

23 Nagino M, Nimura Y, Kamiya J, Kanai M, Hayakawa N, Yamamoto H. Immediate increase in arterial blood flow in embolized hepatic segments after portal vein embolization: CT demonstration. AJR Am J Roentgenol 1998;171(4):1037-1039

24 Kobayashi S, Ng CS, Kazama T, et al. Hemodynamic and morphologic changes after portal vein embolization: differential effects in central and peripheral zones in the liver on multiphasic computed tomography. J Comput Assist Tomogr 2004;28(6):804-810

25 Toni R, Bolondi L, Gaiani S, et al. Accessory ultrasonographic findings in chronic liver disease: diameter of splenic and hepatic arteries, fasting gallbladder volume, and course of left portal vein. J Clin Ultrasound 1985;13(9):611-618

26 Esen K, Balci Y, Tok S, Ucbilek E, Kara E, Kaya O. The evaluation of the right inferior phrenic artery diameter in cirrhotic patients. Jpn J Radiol 2017;35(9):526-531

27 Ramanathan S, Khandelwal N, Kalra N, et al. Correlation of HVPG level with ctp score, MELD Score, ascites, size of varices, and etiology in cirrhotic patients. Saudi J Gastroenterol 2016;22(2):109-115

28 Ozbülbül NI, Yurdakul M, Tola M. Does the right inferior phrenic artery have a supplying role in liver cirrhosis without hepatocellular carcinoma? A 64-slice CT study. Diagn Interv Radiol 2011;17(3):239-242

29 Kim HC, Chung JW, An S, Son KR, Jae HJ, Park JH. Hepatocellular carcinoma: detection of blood supply from the right inferior phrenic artery by the use of multi-detector row CT. J Vasc Interv Radiol 2008;19(11):1551-1557 